\title{
Identification of Aircraft Dynamics Using a SOM and Local Linear Models
}

\author{
Jeongho Cho, Jing Lan, Geetha K. Thampi, Jose C. Principe and *Mark A. Motter \\ Computational NeuroEngineering Lab. \\ University of Florida, Gainesville, FL 32611 \\ * Dynamics and Control Branch \\ NASA Langley Research Center, Hampton, VA 23681 \\ jeongho,principe@cnel.ufl.edu
}

\begin{abstract}
The Self-Organizing Map (SOM) is a powerful tool to produce topology preserving subspace mappings of high dimensional data. In this work we combine a SOM with a set of local linear models to implement functional mappings and identify potentially nonlinear plants. The large dimensionality of the spaces involved (many degrees of freedom and large dynamic range of parameters) is an issue, hence we compare fixed versus growing SOM topologies. The performance of the proposed algorithms $i$ is tested on the simulated data obtained from a realistic aircraft model.
\end{abstract}

\section{Introduction}

The dynamic neural network paradigm provides a promising alternative for the processing of nonlinear signals. For example, feedforward neural networks have been successfully applied to the prediction and modeling of chaotic series, nonlinear filtering, and input-output modeling of nonlinear processes [1]. Due to the universal approximation capability of neural networks in functional spaces of practical importance (finite memory), this method provides a better performance in fitting nonlinear input-output relationships than the polynomial method.

SOM is a topology that converts complex, nonlinear statistical relationships of high-dimensional data into simple geometric relationships [3]. The role of the SOM is to divide the input space into a set of regions represented by the weights of each processing element (PE). However the original SOM is not appropriate for functionals [3].

An alternative modeling methodology is to use local linear models [5]. In this approach, the state space is divided into local regions, where a linear system is used to model the dynamics of each region locally. Since the local modeling uses a divide and conquer approach, it fits each linear system to a segment of the trajectory producing an overall model that may be more accurate when compared to a single global nonlinear model [5]. Besides, when controller design is the target application, the set of local linear models simplifies the design as there is no simple method to design a controller for a nonlinear plant; on the other hand, designing controllers for each linear model can be easily accomplished.

In this paper, a SOM is utilized to cluster the dynamics in state space, as proposed originally by [1]. The standard SOM PEs are extended with local linear models to enable the original algorithm to learn input-output relationships with reasonable accuracy. This is achieved by coupling each PE with a linear mapping in such a way that a functional relationship can be established between each Voronoi region in the input space (of the SOM) and the desired signal. An improved method for function approximation and system identification based on the growing SOM (GSOM) is also developed and compared with the standard SOM. The proposed system identification scheme was tested using data obtained from a realistic aircraft model.

\section{Local Dynamic Modeling}

\subsection{Description of the system}

The LoFLYTE® testbed aircraft designed by Accurate Automation Corporation (AAC) is used to demonstrate the versatility of the proposed algorithm in system identification.

The longitudinal motion consists of axial $(x)$, vertical $(z)$ and pitching $(q)$ motion, while the lateral motion consists of rolling $(p)$, yawing $(r)$ and lateral $(y)$ movement. The elevator surfaces and the throttle control the longitudinal motion, and the aileron and rudder primarily affect lateral motion. The resulting linearized 
equations of motion are sixth-order representing the perturbations in longitudinal and lateral motion. Here, we wish to estimate the aircraft's parameters of longitudinal motion and lateral motion. In general, the dynamics of the system is described by its state dynamic equations and the output mapping. Define

$\vec{x}(k)=\left[\begin{array}{c}p(k), \mathrm{rad} / \mathrm{sec} \\ r(k), \mathrm{rad} / \mathrm{sec} \\ v(k), \text { feet } / \mathrm{sec} \\ q(k), \mathrm{rad} / \mathrm{sec} \\ u(k), \text { feet } / \mathrm{sec} \\ w(k), \text { feet } / \mathrm{sec}\end{array}\right], \vec{u}(k)=\left[\begin{array}{c}\text { aileron angle in degrees } \\ \text { rudder angle in degrees } \\ \text { elevator angle in degrees } \\ \text { throttle }\end{array}\right]$.

Then,

$$
\begin{aligned}
& \vec{x}(k+1)=f(\vec{x}(k), \vec{u}(k)) \\
& \vec{y}(k)=h(\vec{x}(k), \vec{u}(k))
\end{aligned}
$$

\subsection{Local linear models}

In most real-life problems, the dynamic equations describing the system dynamics are unknown. The designer has access to the samples from the input-output data. Therefore the task of dynamic modeling is to find $\tilde{f}$ as an approximation of the functional model $f$ to preserve the same dynamical properties [5]. Assume that a time varying linear model of a signal generating process is given by

$$
y(k)=\vec{a}(k)^{T} \vec{c}(k)
$$

where $\vec{a}(k)=\left[a_{1}(k), \cdots, a_{m}(k)\right]^{T}$ is a set of unknown time-varying parameters and $\vec{c}(k)$ contains the states and control inputs: $[\vec{x}(k), \vec{u}(k)]$

The relation given by (2) could also be considered as linearization of a nonlinear system at the current time $k$. In local linear modeling we use a limited number of local models to approximate the system defined by (2) that is, we are quantizing and limiting the variability of $\vec{a}(k)$ over time. Therefore the modeling problem becomes one of choosing the $\vec{a}(k)$ to describe the dynamics. This is where the SOM is essential for local linear modeling because it creates a clustering of the input dynamics whose centers are associated with a finite number of $\vec{a}(k)$. Moreover, the winner take all mechanism selected at each time step only one of the local models as to approximate the unknown system.

\section{Methodology}

We utilize a SOM to cluster the input signal, constructed through competitive learning such that the trained neural field bears a strong global resemblance to the input space [3]. Therefore the SOM is employed as a modeling infrastructure to construct the local linear model. However, it is difficult to select the size of a regular SOM in advance without any a priori information available, and training may also be difficult.

To overcome these deficiencies of the regular SOM, we also employ the Growing-SOM (GSOM), which has only two major differences from the regular SOM: GSOM uses a constant adaptation parameter for the winning PE and its neighbors. The GSOM adapts only the winning PE and its direct topological neighbors [2].

Our work shows that the dynamics can be modeled by a set of local models, each directly fitted to the quantized state space obtained from the SOM. The proposed nonlinear modeling scenario follows three steps:

1) Embedding the time series

2) Training the SOM

3) Estimation of the local linear models

\subsection{Embedding the time series}

When working with experimental data, we are restricted to observe the system outputs and infer the dynamics from the observation. According to Taken's Embedding Theorem [4], the time delay embedding, which are formed time delayed values of the measurements,

$$
\vec{c}(k)=[\vec{c}(k), \vec{c}(k-\tau), \cdots, \vec{c}(k-(N-1) \tau)]
$$

provides a one-to-one image of the original set, provided the embedding dimension, $N$, is large enough. In MIMO system identification the choice of the embedding (i.e. which inputs and the embedding dimension) plays an important role in defining the representation space.

\subsection{Training of regular SOM}

This step is accomplished via the Kohonen learning process [3]. Let $\overrightarrow{\boldsymbol{c}}(k)$ denote the input vector for the SOM and $w_{i}$ denote the weight vector of PE $i$. With each vector, $\vec{c}(k)$ which consists of $\vec{u}(k)$ and $\vec{x}(k)$ as well as their delayed samples, presented as the input to the network, the Kohonen learning algorithm adaptively discretizes the continuous input space into a set of $\mathrm{K}$ disjoint Voronoi cells. The response of a SOM to input $\vec{c}(k)$ is determined by the reference vector $w_{i^{o}}$ of the PE that produces the best match to the input. Then the $k^{\text {th }}$ adaptation of the weights is done in the following manner.

$\boldsymbol{w}_{i}(k+1)= \begin{cases}\boldsymbol{w}_{i}(k)+\eta(k) \Lambda_{i}(k)\left(\overrightarrow{\boldsymbol{c}}(k)-\boldsymbol{w}_{i}(k)\right), i=i^{o} \\ \boldsymbol{w}_{i}(k), & \text { otherwise }\end{cases}$

where $\eta(k)$ is the learning rate and $\Lambda_{i}(k)$ is a typical neighborhood function. 


\subsection{Training of GSOM}

The initial topology of the network is a triangle. During a self-organizing process, new cells will be added to the map. Every modification of the network is performed such that afterwards the network consists solely of 2-D topology and triangle neighborhood connection again [2].

During training, one adaptation step in our model can be formulated as follows:

1) Locate the winning PE, $s=\boldsymbol{m i n}[\operatorname{dist}(\overrightarrow{\boldsymbol{c}}-\boldsymbol{w})]$

2) Increase matching for $s$ and its direct neighbors $N_{s}$ $\Delta w_{s}=\varepsilon_{b}\left(\overrightarrow{\boldsymbol{c}}-w_{S}\right), \Delta w_{c}=\varepsilon_{n}\left(\overrightarrow{\boldsymbol{c}}-w_{c}\right) \quad\left(\right.$ for all $\left.c \in N_{s}\right)$

3) Increase the signal counter of $s: \Delta \tau_{s}=1$

4) Decrease all signal counter by a fraction

Our objective is a structure with the weights $w_{c}$ distributed according to the distribution of data. With the local signal counters, we can compute an estimate of it, namely the relative frequency of input signals received by a certain cell. When we insert a new PE, it is connected to the others in such a way that we have again a structure consisting only of previous structure. The insertion of $r$ leads to a new Voronoi region $F_{r}$ in the input space. At the same time the Voronoi regions of the topological neighbors of $q$ are diminished.

\subsection{Estimation of the local linear models}

The SOM preserves topological relationships in the input space in such a way that neighboring inputs are mapped to neighboring PEs in the map space. Then, when each PE is extended with a linear model it can actually learn the mapping $y=g(\overrightarrow{\boldsymbol{c}})$ in a supervised way. Each PE has an associated linear model $\vec{a}_{i}$ that represents the linear approximation of the local dynamics.

The linear model weights $\vec{a}_{i}$ are computed directly from the desired signal $y_{i}$ and the clustered inputs by a least square fit within a local neighborhood centered at the current winning PE. The size of the neighborhood must be at least equal to the input space dimension. The design procedure for this linear model is as follows:

1) Apply training data to the SOM and find the winning $\mathrm{PE}$ corresponding to the inputs such that we have winnerinput pairs.

2) Use the least square fit to find the local linear model coefficients for the winning PE where desired output vectors $\vec{y}_{i}^{j}$ within a local neighborhood size of $N_{s}$ as

$$
\vec{y}_{i}^{j}=\vec{a}_{i^{\circ}}{ }^{T} \overrightarrow{\boldsymbol{c}}_{i}^{j}, \quad j=1, \cdots, N_{s}, \quad i=1, \cdots, M_{j}
$$

where $\vec{a}_{i}$ is the sought linear model coefficients, and $\overrightarrow{\boldsymbol{c}}_{i}^{j}$ are the input vectors of the winning PE and input vectors of the $j^{\text {th }}$ closest neighbor to the winner.

3 ) In testing, once the winning PE is determined we select the appropriate linear model from the list of associated models. Apply the local model to obtain the estimated output

$$
\widetilde{\vec{y}}(k)=\vec{a}_{i^{i}} \overrightarrow{\boldsymbol{c}}(k)
$$

\section{Simulation Results}

In this section, we present some simulation results obtained from the application of the proposed method on the identification of a LoFlyte aircraft system.

A total of 1225 PEs, arranged on a $35 \times 35$ square lattice, constitute the SOM neural field. We selected an embedding dimension of $N=3$ for 6-D states and $N=1$ for 4-D control inputs. The embedding dimension is less for control inputs since the change is not drastic between samples. Thus the number of input parameters is 32 . The SOM is trained with 5000 samples for 8000 epochs. The training samples are obtained by exciting the aircraft dynamics using the control inputs.

To explore the usefulness of GSOM for system identification, an experiment is carried out growing a 650 PEs GSOM. All other training conditions are kept the same to have a fair comparison between the regular SOM and GSOM.

The trained systems are tested on a new sequence of 600 samples for all 6-D outputs. The actual and identified trajectory of the system and the corresponding errors using SOM and GSOM, are presented in Figure 1-4. They show that the identified output is pretty close to the actual output except at those points where there is a sudden discontinuity. As can be seen in Table 1, the identification is done with small errors, and the two methods create very similar results.

Table 1. Comparison of $I_{2}$ norm of errors

\begin{tabular}{|c|c|c|c|c|c|c|}
\hline & $p$ & $q$ & $r$ & $u$ & $v$ & $w$ \\
\hline \hline Regular SOM & 0.19 & 0.07 & 0.02 & 0.02 & 0.01 & 0.03 \\
\hline GSOM & 0.44 & 0.10 & 0.02 & 0.02 & 0.02 & 0.03 \\
\hline
\end{tabular}

However, since the GSOM uses almost half the number of PEs of a regular SOM to identify the system GSOM is a better modeling structure than a regular SOM. The main advantage of GSOM is that the network structure is determined automatically from the input data. Compared with a regular SOM, GSOM has all its PEs within the distribution envelope of training data. Also, after training, all PEs are either a winner or winner's. However, if there is some testing data that is out of the range of training data, both the winner and its neighbors 
have a large distance to the data. It leads to occasional large errors in the prediction results shown in Figure 2.
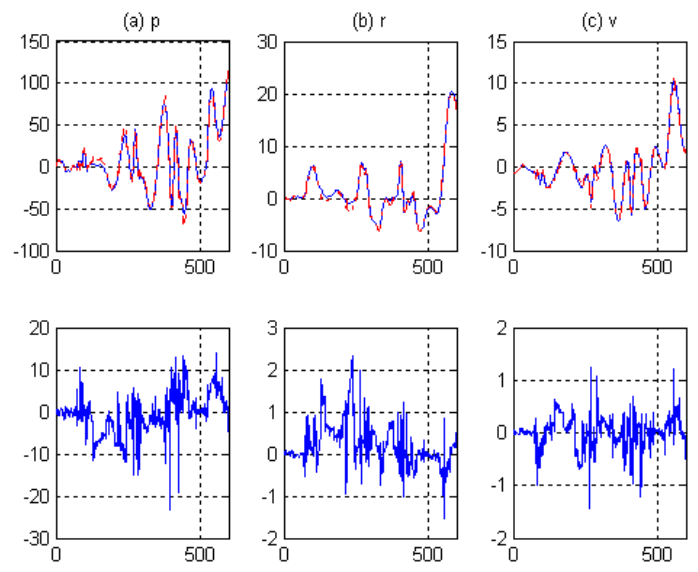

Figure 1. Parameter estimation of lateral motion using regular SOM.
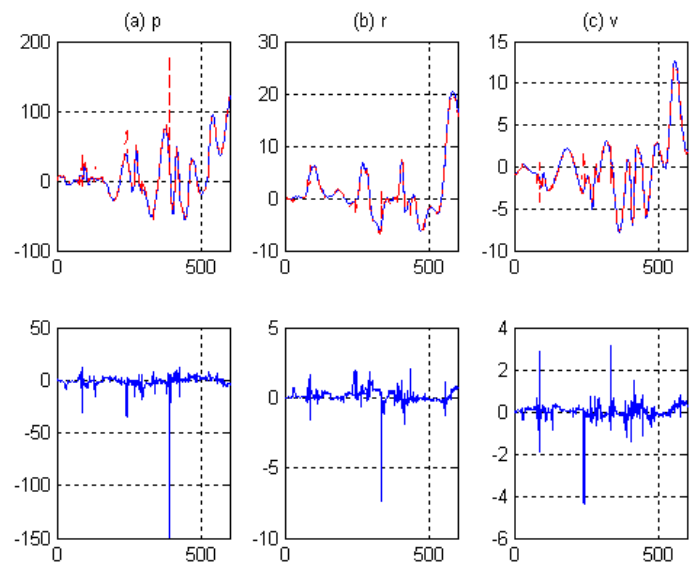

Figure 2. Parameter estimation of lateral motion using GSOM.
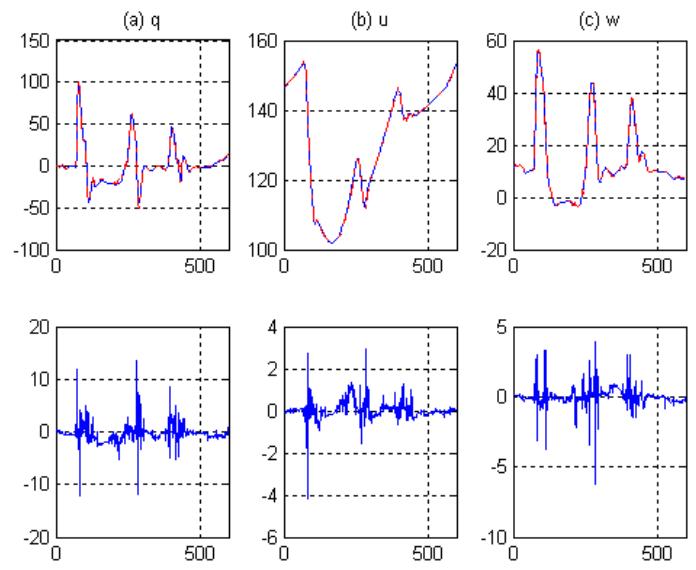

Figure 3. Parameter estimation of longitudinal motion using regular SOM.
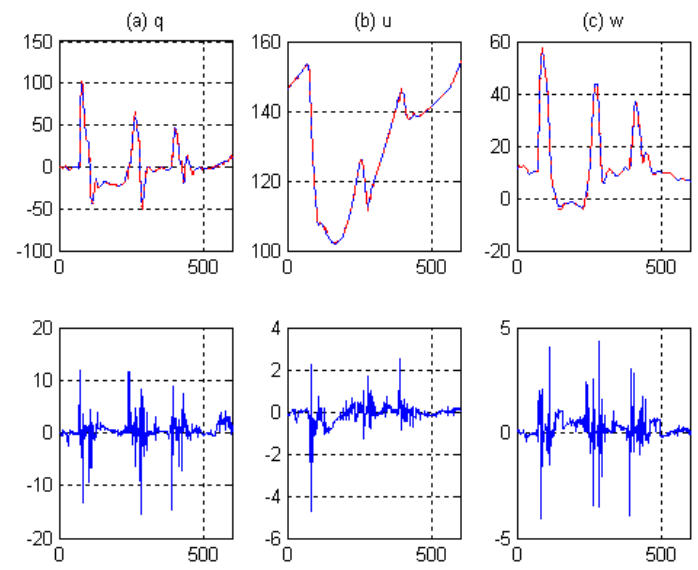

\section{Figure 4. Parameter estimation of longitudinal motion using GSOM.}

\section{Conclusion}

There has been an increased interest on multiple models for numerous engineering applications. In this paper, we have combined the superior clustering capability of SOM with the simplicity of local linear modeling for system identification. The proposed architecture involves a SOM that determines the region where the state dynamics of the observed system is and selects an appropriate local linear model. The selected linear model, which is optimized to represent the local dynamics, is then used to produce an estimate of the system output.

The obtained set of local linear models can be used in controller design problems. The application we have presented is specific to the control of an experimental aircraft. In simulations, we have verified that the SOM successfully quantizes the state space and the linear models produce promising results.

Acknowledgements: This work was partially supported by Accurate Automation Corporation under grant \#463, and NASA grant NAG-1-02068.

\section{References}

[1] J.C. Principe, L. Wang, and M.A. Motter, "Local Dynamic Modeling with Self-Organizing Maps and Application to Nonlinear System Identification and Control," Proc. of IEEE, Vol. 86, No. 11, 1998, pp. 2240-2258.

[2] B. Fritzke, "Growing Cell Structures - A Self-Organizing Network for Unsupervised and Supervised Learning," IEEE Trans. Neural Networks, Vol. 7, No. 9, 1994, pp. 1441-1460.

[3] Kohonen, T., Self-Organizing Maps, Springer, 1995.

[4] F. Takens, "Detecting strange attractors inturbulence," in Dynamical Systems and Turbulence (Springer Lecture Notes in Mathematics), Vol. 898, 1980, pp. 365-381.

[5] M. Casdagli, "Nonlinear prediction of chaotic time series," Phys. D, Vol. 35, 1991, pp.27 